\title{
Usability Evaluation Criteria for Internet of Things
}

\author{
Michael Onuoha Thomas \\ Asia Pacific University of Technology \& Innovation (APU), \\ Technology Park Malaysia, Bukit Jalil, 57000 Kuala Lumpur, Malaysia. \\ E-mail: onuohathomas@gmail.com \\ Beverly Amunga Onyimbo and Rajas varan Loges waran \\ Asia Pacific University of Technology \& Innovation (APU), \\ Technology Park Malaysia, Bukit Jalil, 57000 Kuala Lumpur, Malaysia. \\ E-mail:beverlyamunga@g mail.com, logeswaran@apu.edu.my
}

\begin{abstract}
The field of usability, user experience (UX) design and human-computer interaction (HCI) arose in the realm of desktop computers and applications. The current experience in computing has radically evolved into ubiquitous computing over the preceding years. Interactions these days take place on different devices: mobile phones, e-readers and smart TVs, amid numerous smart devices. The use of one service across multiple devices is, at present, common with different form factors Academic researchers are still try ing to figure out the best design techniques for new devices and experiences. The Internet of Things (IoT) is growing, with an ever wider range of daily objects acquiring connectivity, sensing ability and increased computing power. Designing for IoT raises a lot of challenges; the obvious difference being the much wider variety of device form factors. IoT is still a technically driven field, thus the usability of many of IoT products is, in some way, of the level anticipated of mature consumer products. This study focuses on proposing a usability evaluation criterion for the generic IoT architecture and essential technological components.
\end{abstract}

Index Terms-Internet of Things (IoT), Usability evaluation, Devices, Quality, Systems.

\section{INTRODUCTION}

The Internet of Things (IoT) is the future of the Internet and an integrated part of the modern person's daily life. Statistics and trends in the field of Information Technology (IT) indicate that the Internet is now literally used for everything and by everyone. IoT systems are perceived to support various spectrums of users, technical and non-technical, in the same way [1]. The encroachment of ubiquitous computing technologies, like wireless networks and mobile devices, partake significantly in the augmented availability of digital information and services in our day to day lives, transforming how people access and make use of them. The IoT is a technology that spreads digital resources to the real world, linking such resources with daily objects by augmenting the latter with RFID (Radio Frequency Identification) or Near Field Communication (NFC) tags.

Usability has been increasingly recognized as a crucial quality dimension to arbitrate the success of interactive systems. According to [2], usability is "the competency of a product to be understood, learned, operated and is attractive to the users' when they are used to achieving certain goals with effectiveness and efficiency in specific environments". The authors further expound that the usability of a product is habitually validated through its interfaces.

To date, there are no defined usability dimensions or guidelines specifically meant for IoT devices. The guidelines available are particularly intended for desktop and web based applications and systems. The IoT tends to use mobile applications, in addition to web based and desktop applications, due to their mobility nature. To ensure that systems meet their expected quality performance, a number of usability guide lines have been introduced in the past [38]. These, however, are only generic rules that guide the design and implementation for the IoT. Usability guidelines in this field are lacking as usability is relatively unexplored and unproven [3].

This study aims to address this is sue by proposing a set of usability dimensions to be considered in the design and evaluation of IoT systems. This research also intends to review the existing usability guidelines for IoT systems, in order to identify and prioritize the usability dimensions based on its importance. The guidelines and usability evaluation criteria are proposed based on reviews of previous related studies and observations of current trends. The findings of this work could motivate the initial steps in the development and introduction of more targeted guidelines for IoT systems.

This paper is organized as follows: this section provides a brief introduction on the meaning of usability and IoT. Next, section II explores related works in IoT as well as usability, to gain insight on how they are intertwined. Section III gives an in-depth review of IoT, its applicability and technological components. Section IV then explores the aspect of usability engineering and important usability evaluation criteria, as well as 
important requirements necessitated for usability integration. Finally, a conclusion of the general overview of the research and recommendations for the proposed evaluation criteria are discussed in section $\mathrm{V}$.

\section{RELATED WORKS}

The principle and concept of internet usage have witnessed a new revolution with exponential changes in operational abilities [4]. This change has significant effect and impact on every aspect of our lives, and serves as a new frontier towards future evolution of technology. [5] explains that the technological concept of IoT revolves around interconnectivity between objects within our surroundings; this is applicable to all scenarios of our lives, e.g. wearables, smart phones and TV's etc. According to [6], the operational abilities of these smart devices should be easy and smooth for the users, and must function maximally at all times. Although the technological concept of IoT presents great opportunities, according to [7] and [8], there are still many open challenges that must be addressed to ensure smooth operability and applicability.

There are currently no standardization and quality dimensions for IoT applications and devices [7], [9]. As such, [10] proposes a quality dimensional model, which must address issues related to usability, operability, reliability, responsiveness and personalization. The application of usability engineering process in the development of IoT device interface would ensure smooth and easy to use operational systems for the users. Integrating usability prospects into the development of IoT devices also provides reliability assurance for the user based on the functionality of the system or device.

\section{INTERNET OF THINGS (IOT)}

This technological paradig $m$ has over the years become a focal area of research at both the industrial and academic levels. The concept, which introduces a new era in computing, gives inanimate objects the capability of communicat ing. The term 'Internet of Things' (or IoT, as it is popularly abbreviated) was invented by Kevin Ashton in 1999, in the context of supply chain management [5]. In both the industrial and academic scenario, IoT has been defined in different aspects and scenarios depending on its application. The IoT, as stated by [11], is a technological advancement which is recognized as one of the most important aspect of future technology, with a forecast that this technology would evolve beyond 26 billion units by 2020 (up from 0.9 billion in 2009).

According to [12], the IoT paradigm is based on "intelligent and self-configuring nodes (things) interconnected in a dynamic and global network of infrastructure". It provides connectivity for anyone and anything at any given point in time. The basic technological concept behind this paradigm, according to [13], is to give autonomous and secure connectivity between objects (i.e. things) with processing capability through exchange of data amongst real world devices and applications. It is characterized with several features comprising of the complex working environment, wide distribution of network segment, as well as a no specific standardized network topology [14]. Some basic technological components towards making the vision for connected objects a reality include radio frequency identification (RFID), sensors, actuators, mobile phones and other portable devices.

\section{A. Internet of Things (IoT) Scenario}

As a technological paradigm with heterogeneous components, the IoT has over the years been inevitably applied and integrated into different scenarios in the modern environment [15]. According to [8], the IoT embraces the convergence of different technological components for sensing, connectivity, processing, as well as control, through application software. It enables vast and sophisticated service processing for tracking, composed of heterogeneous devices by creating communication channels and also translating their functionality into useful service for the user. Examples of major application domains are described as depicted in Fig. 1.

Smart homes: According to [15], home and office automation has been made possible due to technological advancement in the IoT as it decreases consumption of resources associated to building, e.g. water and electricity, and also improves the standard of living for humans. Sensors are constantly integrated into homes and office equipment for easy monitoring of resources and users' needs [8]. A notable instance of automation in homes and office includes internet enabled television control, air conditioning automation, switching on/off of lighting, etc.

Transportation: As suggested in [16], a notable application area of IoT technology in transportation is in the vehicle anti-theft tracking system. This technology gives the user the ability to comfortably monitor transitions of the stolen vehicle with options for visualizing the process through a GPS software- enabled application. Smart transportation enables logistical tracking of assets irrespective of its location and environment, and also provides an avenue in which these assets communicate with the users through chips embedded in them [17].

Healthcare: This is an important domain in which the IoT actively enhances productivity and improves service delivery [5], [18]-[20]. It provides a systematic mechanis $m$ in which track records of human health can be analyzed, monitored and also provide emergency services to individuals in need as a result of actively tracking the health activity of the individual. In hospitals, RFID technology (an important driving force in the IoT) has been actively deployed into medical equipment for easy tracking. 


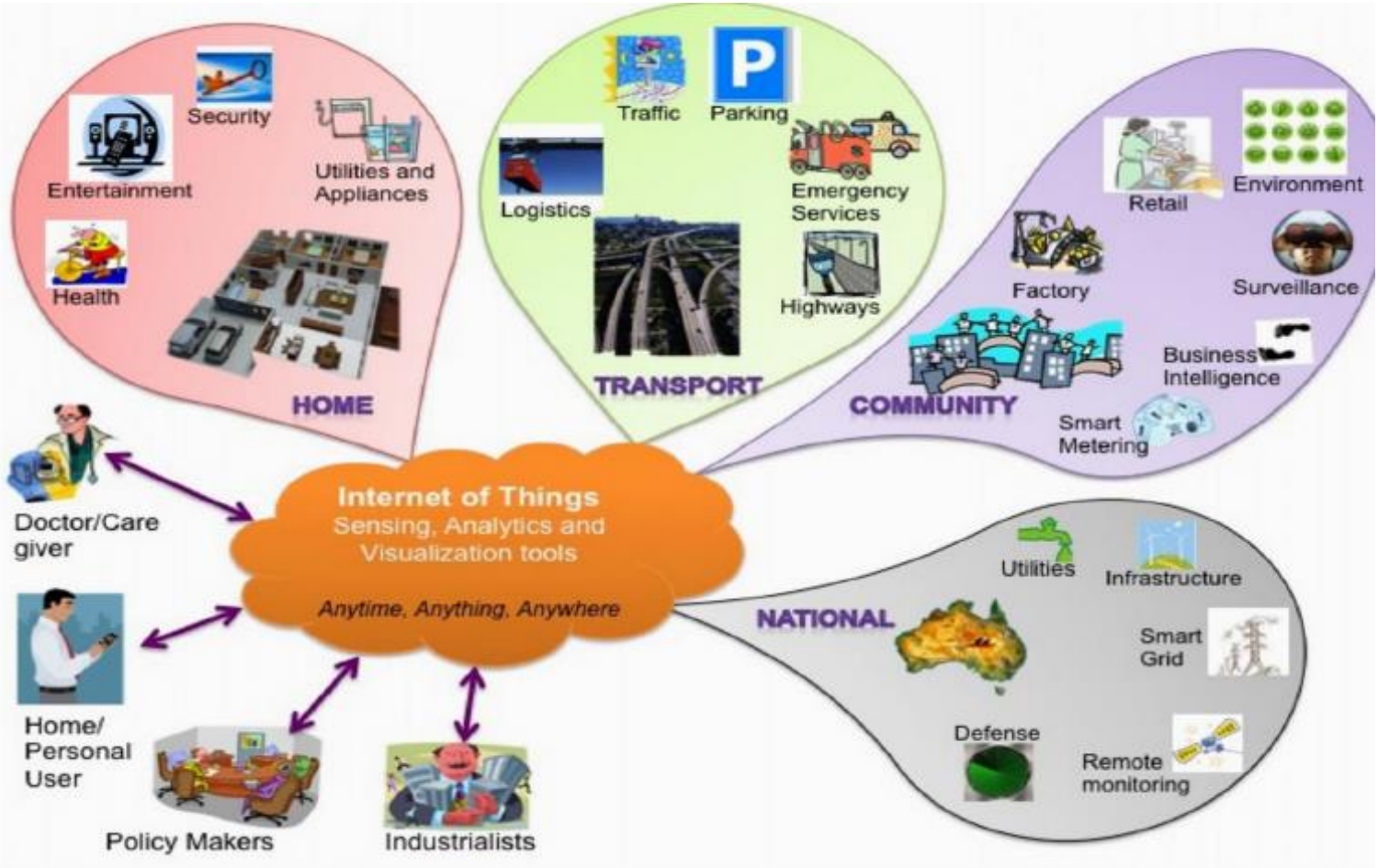

Fig.1. End-user application scenarios of IoT [4]

\section{B. Internet of Things (IoT) Architecture}

Research in [7]-[8] and [18]-[20] suggest that the IoT is composed of four fundamental layers: perception, network, middleware and application, as described below:

- Perception layer:

In [13], this layer is described as the device layer. It constitutes the physical objects as well as the microprocess or chips and sensors. It comprises of the RFID tags, sensors, bar-codes, bluetooth, infrared technology, etc. This layer deals with giving identity to objects as well as collecting of identity and vital data through the sensors, which are passed to the network layer to provide secure data processing and transmis sion.

\section{- Network layer:}

Generally characterized as the fundamental enabling platform for communication between devices in the IoT paradigm, which relates and transmits objects (things) status and data [24]. It consists of both wired and wireless access communications, commonly using the cellular, WIFI, microwave and satellite infrastructures.

- Middleware layer:

This basically comprises of information gathering and intelligent processing. It bridges the gap between the eccentric devices, which include software control components of the IoT, cloud management platform, data centers and control centers [25].

- Application layer:

This layer is characterized as the application support that enables developers to easily carryout authentication and certification of device management for the end users.

\section{Essential Technological Components of IoT}

According to [5]-[6], [19]-[20] and [22], the following are the essential components that make the IoT paradig $\mathrm{m}$ a possibility:

- $\quad$ Radio Frequency Identification (RFID):

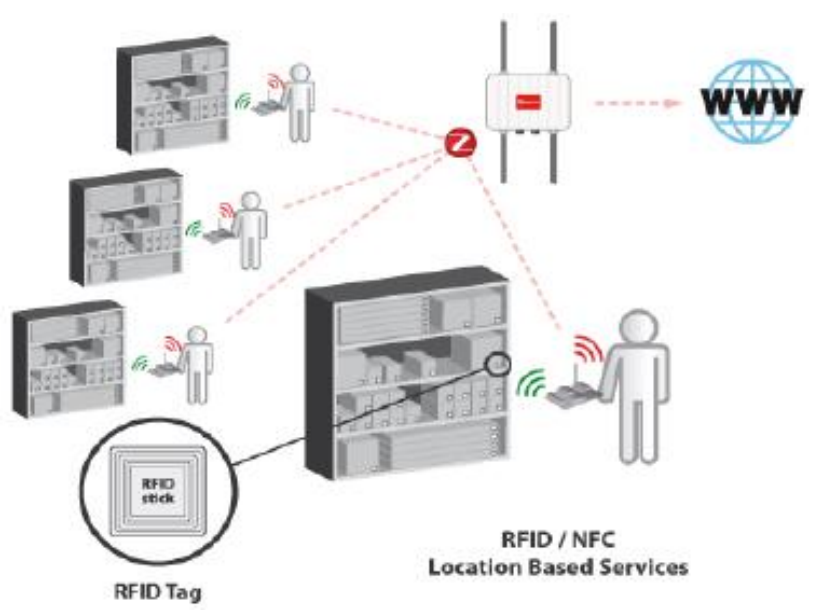

Fig.2.Schematic representation of RFID [52]

This technological component is characterized as one of the enabling components of the IoT, as described in [11]. It allows for autonomous identification of objects (things) as well as data capture of fundamental information about the objects through radio waves, tags and a reader. Fig. 2 shows a schematic representation of 
RFID. These sensor-like chips provide identity to anything they are attached to and also enable integration of objects into a broad network domain [12], [21]. This principle serves as a pivotal enabler for the technological component to continuously acquire status information about its environment. They play an important role towards linking the gap between the physical world and the information processing world. According to [5] and [18], there are three types of RFID tags:

(1) Active RFID tags - this type of RFID tags is battery-enabled with the capability of efficiently communicating with the reader. The energy supplied to the tag helps to init iate communication with the reader.

(2) Passive RFID tags - this type of tags solely depends on the radio frequency energy that is transferred from the reader to the tag, for power and communication.

(3) Semi-passive RFID tags - This type of tags has embedded batteries that powers the mic rochips for communication through powers from the reader.

\section{- Wireless Sens or Network (WSN):}

This technological component of the IoT allows for different network topologies and multi-hop communication between embedded objects. It consists of spatially autonomous distribution of sensors / RFID enabled devices that continuously monitor environmental as well as physical conditions of basic status, locations or transitions in embedded objects [11], [27]. A WSN architectural representation is given in Fig. 3.

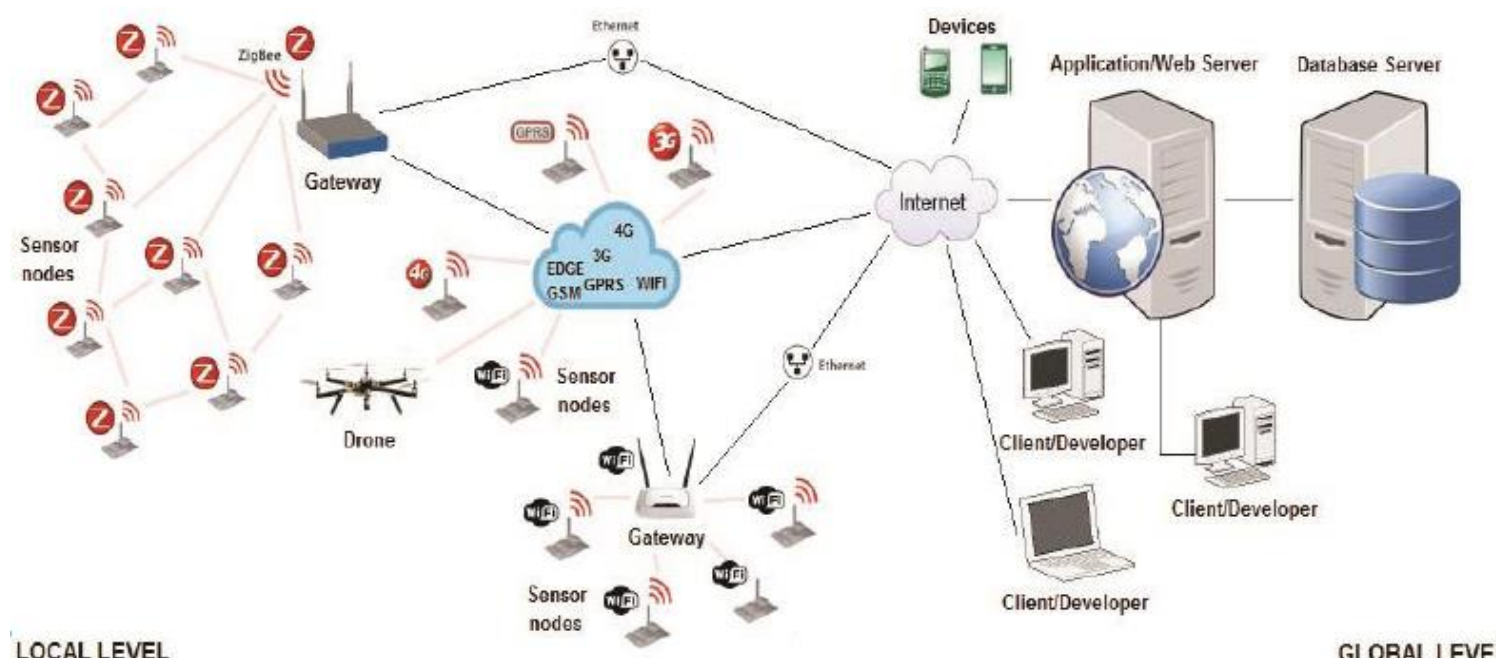

Fig.3. Architectural representation of WSN [27]

\section{- Middleware:}

It is a mechanism at which the cyber infrastructure, service oriented architecture and sensor network interpose, in order to provide access towards the IoT heterogeneous sensor resources [5]. It is based on the concept of isolating resources that can be utilized by different software applications. It is characterized as a software layer [11] in the IoT paradig $\mathrm{m}$, which interposes between the functional control of the software applications and enables communications of the basic input and output of data.

\section{- Cloud computing:}

As described in [28], cloud computing creates enabling ways in which the IoT systems are designed, developed, tested, deployed and maintained on the Internet. It applies a utility model that defines how embedded systems consume computing resources (e.g. storage).

Cloud computing is defined as a technological concept that provides huge data streams for storage capabilities to everything or anything with processing capability [29],
[30]. It is considered as a complex system of distributed parallel computing, utilizing computing with network and virtualization technology. It serves as a back-bone of the IoT because of its capability to provide back-end solutions towards handling of the enormous data streams emanating from various IoT devices. Analyzing the technological concept of the IoT [11], [31], many of its applications demand enormous amounts of data storage, high processing speed in order to enable real-time communication, as well as easy decision making processing. Cloud computing for the IoT is capable of providing on-demand access to a shared pool of configurable resources and devices, i.e. networks, servers, wearables, storage and applications, to meet the required needs. An example of an end-to-end model of interaction in a cloud centric IoT paradigm is given in Fig. 4.

\section{- Application software:}

The application software of the IoT is considered as a platform for communication. It enables easy communication between machine-to-machine and human-to-machine. 


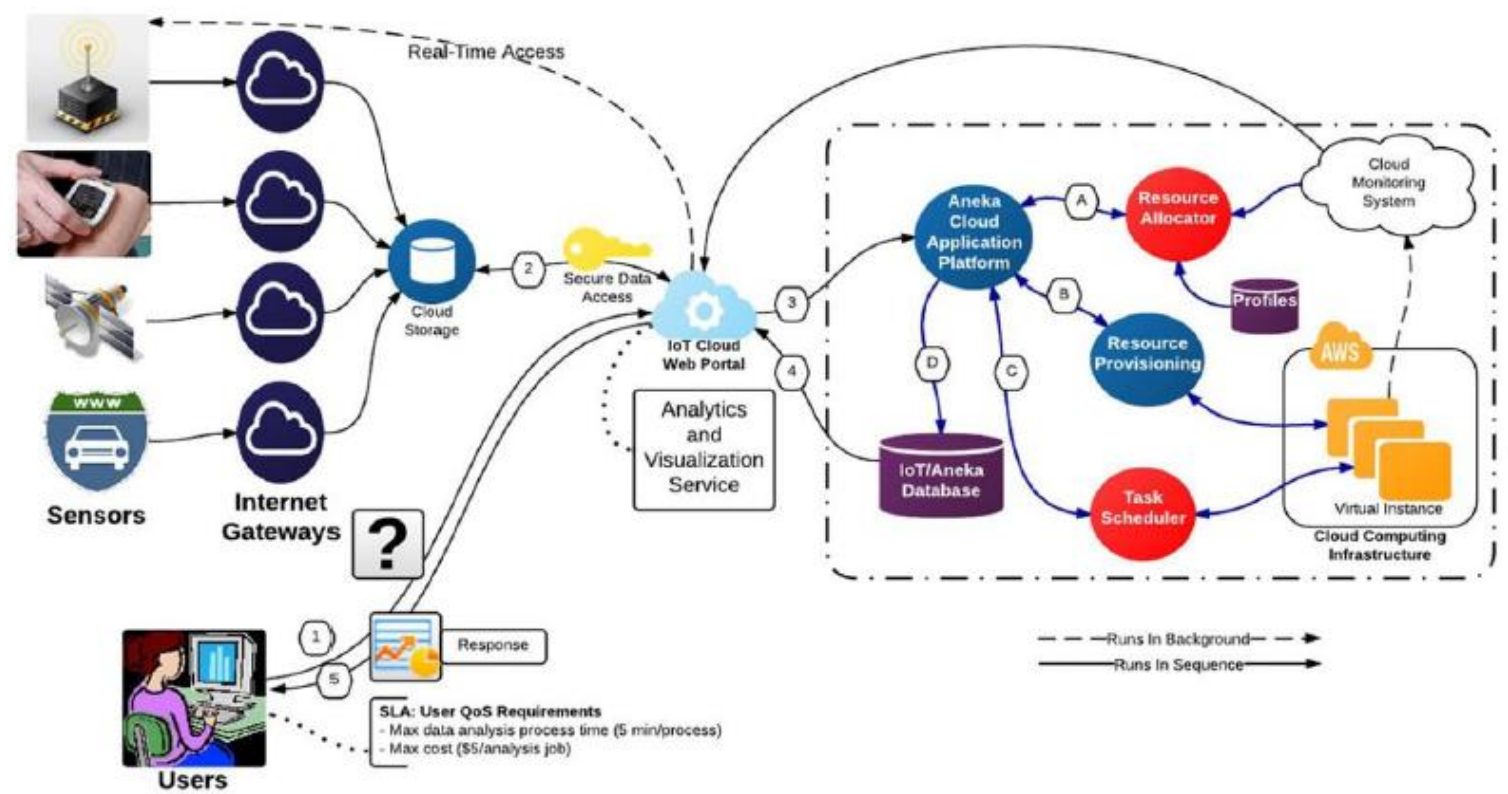

Fig.4. An end-to-end model of interaction in cloud centric IoT paradigm [5]

A notable example of application software of the IoT scenario is the GPS tracking software for transportation. This software provides a platform for interaction, monitoring and communication between objects in transition, i.e. monitoring of as sets and cartransition.

\section{USABILITY ENGINEERING}

Usability engineering is the branch of engineering concerned with the design and development of systems interface with high usability, a need that arose due to the emergence of complex and sophisticated systems with more interactive interfaces and a wide range of ine xperienced users [32], [33]. The usability engineering process or techniques are geared towards improving existing or intended systems, and is considered as one of the most important focus areas during development, as noted in [34]. It is also characterized as an important quality attribute that every system intended for humans must adhere to, i.e. enabling a user-friendly operational system.

As described in [35], a good user-friendly system is determined in accordance with the objective of the system. This is in accordance with the ISO 9241-11, which defines usability as "the extent at which a product can be used by specified users in achieving specified goals with efficiency, effectiveness and also satisfaction in the specified context of use", and "the quality which characterizes the functional use of a program and application" [36]. Fig. 5 describes the relationship between the important components taking part in the process of usability.

Applying the principles of usability engineering criteria, process or technique, according to [32], [36][38], ensures that every system conforms to specification s and are fit for purpose based on any particular group of user(s) for which the system is created. It also should compose of a broad range of easy to use functionalities.

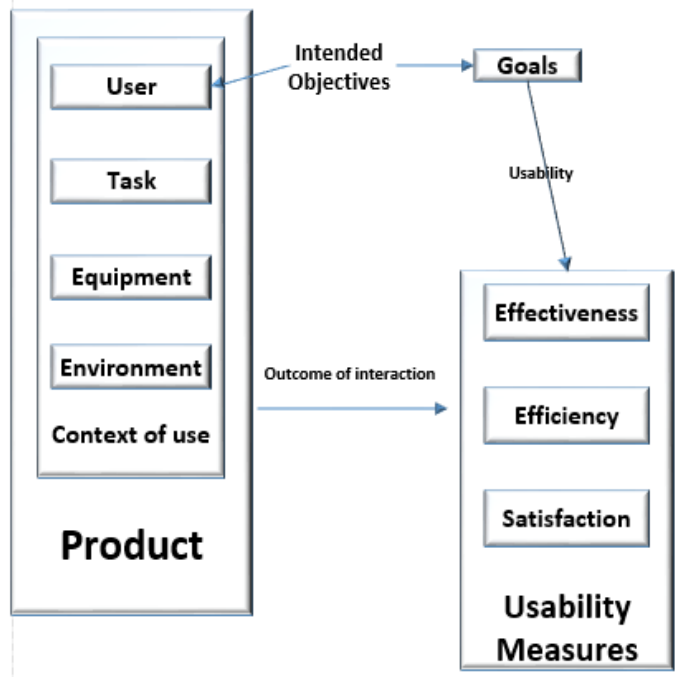

Fig.5. Usability framework [36]

A. Usability Evaluation Criteria for IoT

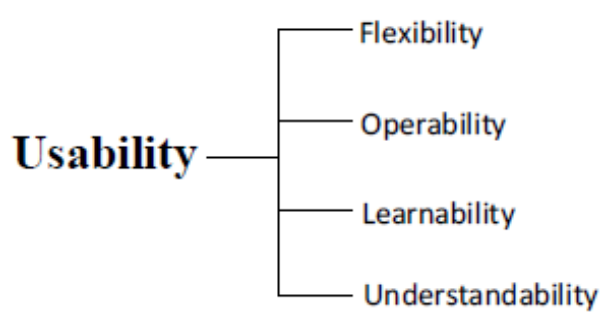

Fig.6. Usability evaluation criteria [9], [35]-[36]

Generally, the usability criteria serves as a basis for determining the ease of use of a computing system. It is 
geared towards ensuring efficiency, effectiveness and satisfaction of computing systems and devices for the users. The criteria shown in Fig. 6 represents the basic fundamental criteria all computing interactive systems or devices must possess for assurance of ease in usage for the users [9], [36]. According to [9], these are also applicable to IoT.

\section{1) Flexibility:}

This characteristic describes the generalized abstraction between various IoT application logic with the system interface [35]. An IoT system is characterized as a heterogeneous system, therefore its components should have the ability to be integrated or incorporated into a new environment without any disruption of service to the user. In every computing system, it is expected that they proffer different ways of executing the same task [36], in order to enable users to choose and adapt to the most suitable functionality based on preference and necessity.

\section{2) Operability:}

Since IoT consists of interconnected heterogeneous components, it is pertinent that they all function towards achievement of users specification [39]-[40]. It is expected that there is consistency in functionality, to enable the users of these systems or devices to become familiar with important functional commands. The adoption of important usability standards, according to [36], would help prevent complications in navigation of the userinterface of IoT devices.

\section{3) Learnability:}

According to [41], the ease of learning of any given system is a major quality characteristic every system should possess. In order to achieve this characteristic, [32] and [42] state that the system should have task(s) that meet the users' way of life. It ensures that bogus technical terms, elements or icons that are not familiar to the user are minimal - this principle ensures that there is no misinterpretation of the functionality to avoid leading the users astray. It is expected that an IoT system or component prevents wrong conclusions, irrelevant contents, and minimizes the use of complex commands by allowing as few as possible actions or functions to perform a task. As noted in [36], complex tasks hinder learning and increases the possibility of errors.

\section{4) Understandability:}

It is generally expected that every system works consistently in terms of functionality, as this enables the user to familiarize with the basic fundamental composition of the system [36]. Functionality, as depicted in [35], describes the quality of an IoT system to be designed, developed and deployed to serve its purposes well in the prescribed duration for which it was manufactured. Considering the dynamic nature of our environment, it is expected that all IoT systems and devices are easy to learn based on the deployment environment [7], [15]. To achieve ease of usage and learnability, IoT systems or devices must be free from ambiguous technical terms or commands that are not widely known by the users [36], [43]. This is to avoid misinterpretation of functionality that might lead to adverse damage.

\section{B. Achieving Usability Criteria in IoT Systems}

From the literature on the IoT and basic usability criteria, the scholars in [16] and [32] suggest that to achieve the basic fundamental usability criteria in a system, some requirements must be considered when integrating the basic usability criteria development and deployment of IoT systems or devices. It is necessitated considering the dynamic nature of our surroundings and due to the fact that the user's perspective and satisfaction about the system is important. The rationale towards requirement specification is to keep the basic fundamental stakeholders in the IoT technological paradigm focused on the goals or purpose of designing or developing the system. These requirements serve as prerequisites for quality assurance or as a checklist to ensure that the basic functionality of a system is strictly adhered to based on the users' needs and preferences.

According to [13], [34]-[36], [38], in deploying any system (e.g., IoT), user's require ments, data require ments, functional requirements, and environmental requirements must be taken into account. These are elaborated below.

\section{1) Data Requirements}

According to [19], "internet of things is a structure in which objects and people are provided with unique identities with capabilities of relocating any data through a network without requiring any two-way handshaking between human-to-human, i.e. source destination or human-to-systems interaction". The IoT is a system that has heterogeneously connected components with many functional and non-functional components and subcomponents. These heterogeneous components constantly transmit data about their location and status [31], [44]. The data transmitted requires enormous amounts of data storage. As a necessity, the data require ment of any proposed IoT device or system should be an essential factor to be deliberated to ensure that the usability of the system is properly addressed and prevents loss of data during transmission. Data loss causes disruption in service, which can be frustrating on the part of the user [45].

Data requirements assists the developers in comprehending how the data will be collected and used, for their planning and development of the database(s) that has a functionality that is in support with the information flow. During data / in formation gathering, it is imperative to have awareness of the storage procedure and format the information / data, for it to be utilized efficiently as well as in several ways [46].

\section{2) Environmental Requirements}

According to [4], the development of a trusted, secure, reliable, interoperable usability criteria into the computing environment of the IoT requires technologies 
that would ensure flexibility and scalability of the system, which gives privilege to the system as IoT with diverse components and a robust operational environment for its processes. As discussed in [4], the IoT system operates around us and are sometimes attached to the human body. This requires its operational capabilities to fall within the environment to which it would be deployed. In [22], it is necessary for environmental factors to be considered to necessitate deployment of reliable systems to the users' environment, with environmental parameters like humidity, temperature and illuminance determined to ensure that the system falls within the boundaries it was created for. According to [47], the system's operational environment determines its operability; when a built system fails to withstand its environmental component, failure occurs that might create panic or discomfort among the users.

\section{3) Functional Requirements}

In order to support users with the presence of a list of features, it is necessary to give a description of their functionality. Workflow is the sequence of steps that the users ought to take in order for them to complete a task. Determining the interaction between one feature and another is also vital because most times, the interaction between the features (two or more) may possibly generate new design problems that are related to functionality. According to [1] and [47], the IoT consists of basic intelligent devices (i.e. sensors), embedded processors and many forms of connectivity components. These components are considered as functional elements, which enable business and human intelligence. The transmitted data from sensors and other intelligent objects serve as an input considered during various decision making for users as well as businesses and customer management [1], [48]. In [50], the functionality of a system determines user acceptance. Through benchmarking, the fundamental functional requirement of a system is enforced; it enables the IoT system functions to be based on specifications and does only what it is intended or built for.

\section{4) User Requirements}

This defines how the physical and cognitive needs of the envisioned users of the system are met. It is vital for users to have the ability to contentedly and effectively make use of an interface to realize the goals it has been designed to support. User requirements can be specified when the users of the system interface and the environment in which it will be used are clearly defined. According to [38], with regards to usability engineering, improving the usability of a system requires a standard benchmark for design, development, implementation and deployment. In [23], it was suggested that a draft of a technical oriented reference document is provided with the aim of representing the standardization of requirements for IoT systems or applications.

A contextual task analysis method can be used to achieve insights on the expectations of the interface use by keenly scrutinizing the way they presently carry out tasks that are similar to what the interface will support. In the situation where the number of the targeted users with disabilities (e.g. impaired $\mathrm{v}$ ision, hearing problems or limited motor skills) is high, one may be forced to come up with an interface design that supports accessibility tools. According to [20], the IoT should have unique features and diverse requirements, be time tolerant, have secure connections, good monitoring and low energy consumption. These service requirements ensure that the user utilizes the system's full potential without dis ruption in service. The reliability of the IoT system provides trust and confidence during usage [9], [51].

\section{CONCLUSION}

As the technological trends continue to expand, especially so for user-enabled technologies, the need to continually implement standards to ensure that systems comply with specifications is required. The IoT technological paradig $\mathrm{m}$ has over the years become a worldwide phenomenon with most of them composed of user interfaces, e.g. smart watches and applications control points in smart phones. Usability engineering processes ensure that certain standards are met and guidelines adhered to, because the likelihood of affecting the user's perspective, way of life and feelings is high.

The usability evaluation criteria, as discussed in this paper, reflects previous literature on the basic fundamental criteria that all intelligent computing systems and devices must possess, including the IoT. In order to properly implement these criteria into the technological paradigm of the IoT, the stated requirements should be met.

\section{REFERENCES}

[1] A. J. Choi, "Internet of Things: Evolution towards a Hyper-Connected Society," 2014.

[2] R. Baharuddin, D. Singh, and R. Razali, "Usability dimensions for mobile applications-a review," Res. J. Appl. Sci. Eng. Technol., vol. 5, no. 6, pp. 2225-2231, 2013.

[3] R. Yáñez Gómez, D. Cascado Caballero, and J.-L. Sevillano, "Heuristic evaluation on mobile interfaces: a new checklist.," ScientificWorldJournal., vol. 2014, pp. 1-20, 2014.

[4] R. D. Sriram and A. Sheth, "Internet of Things Perspectives," IT Prof., vol. 17, no. 3, pp.60-63, 2015.

[5] J. Gubbi, R. Buyya, S. Marusic, and M. Palaniswami, "Internet of Things (IoT): A vision, architectural elements, and future directions," Futur. Gener. Comput. Syst., vol. 29, no. 7, pp. 1645-1660, 2013.

[6] R. Baharuddin, D. Singh, and R. Razali, "Usability dimensions for mobile applications-a review," Res. J. Appl. Sci. Eng. Technol., vol. 5, no. 6, pp. 2225-2231, 2013.

[7] L. Atzori, A. Iera, and G. Morabito, "The Internet of Things: A survey," Comput. Networks, vol. 54, no. 15, pp. 2787-2805, 2010.

[8] D. Miorandi, S. Sicari, F. De Pellegrini, and I. Chlamtac, "Internet of things: Vision, applications and research challenges," Ad Hoc Networks, vol. 10, no. 7, pp. 14971516, 2012.

[9] T. Frühwirth, L. Krammer, and W. Kastner, 
"Dependability demands and state of the art in the internet of things," IEEE Int. Conf. Emerg. Technol. Fact. Autom. ETFA, vol. 2015-Octob, 2015.

[10] P. Martin and K. Brohman, "CLOUDQUAL: A Quality Model for Cloud Services," IEEE Trans. Ind. Informatics, vol. 10, no. 2, pp. 1527-1536, 2014.

[11] I. Lee and K. Lee, "The Internet of Things (IoT): Applications, investments, and challenges for enterprises," Bus. Horiz., vol. 58, no. 4, pp. 431-440, 2015.

[12] A. Botta, W. de Donato, V. Persico, and A. Pescapé, "Integration of Cloud computing and Internet of Things: A survey," Futur. Gener. Comput. Syst., vol. 56, pp. 684700, 2014.

[13] R. Khan, S. U. Khan, R. Zaheer, and S. Khan, "Future internet: The internet of things architecture, possible applications and key challenges," Proc. - 10th Int. Conf. Front. Inf. Technol. FIT 2012, pp. 257-260, 2012.

[14] L. Yong-Fei and T. Li-Qin, "Comprehensive Evaluation Method of Reliability of Internet of Things," 2014 Ninth Int. Conf. P2P, Parallel, Grid, Cloud Internet Comput., pp. 262-266, 2014.

[15] L. Coetzee and J. Eksteen, "The Internet of Things Promise for the Future? An Introduction," Conf. Proc., pp 978-1, 2011.

[16] Z. Liu, A. Zhang, and S. Li, "Vehicle anti-theft tracking system based on Internet of things," Proc. 2013 IEEE Int. Conf. Veh. Electron. Saf., pp. 48-52, 2013.

[17] I. M. Almomani, N. Y. Alkhalil, E. M. Ahmad, and R. M. Jodeh, "Ubiquitous GPS vehicle tracking and management system," 2011 IEEE Jordan Conf. Appl. Electr. Eng. Comput. Technol. AEECT 2011, 2011.

[18] C. Rahmani, A. Azadmanesh, and H. Siy, "ArchitectureBased Reliability Modeling of Web Services Using Petri Nets," 2010 IEEE 12th Int. Symp. High Assur. Syst. Eng., pp. 164-165, 2010.

[19] A. W. Burange and H. D. Misalkar, "Review of Internet of Things in development of smart cities with data management \& privacy," Conf. Proceeding - 2015 Int. Conf. Adv. Comput. Eng. Appl. ICACEA 2015, pp. 189$195,2015$.

[20] M. De Sanctis, E. Cianca, G. Araniti, I. Bisio, and R. Prasad, "Internet of Remote Things," vol. 3, no. 1, pp. 113-123, 2016.

[21] L. Tan, "Future internet: The Internet of Things," 2010 3rd Int. Conf. Adv. Comput. Theory Eng., pp. V5-376V5-380, 2010.

[22] S. Bin, Z. Guiqing, W. Shaolin, and W. Dong, "The development of management system for Building Equipment Internet of Things," 2011 IEEE 3rd Int. Conf. Commun. Softw. Networks, pp. 423-427, 2011.

[23] W. Pollard, "Internet of Things EUROPEAN R ESEARCH C LUSTER ON THE INTERNET OF THINGS," 2015.

[24] J. Rui and S. Danpeng, "Architecture Design of the Internet of Things Based on Cloud Computing," 2015 Seventh Int. Conf. Meas. Technol. Mechatronics Autom., pp. 206-209, 2015.

[25] S. Frost, "Internet of Things," vol. 07, 2015.

[26] X. Li, R. Lu, X. Liang, X. Shen, J. Chen, and X. Lin, "Smart community: An internet of things application," IEEE Commun. Mag., vol. 49, no. 11, pp.68-75, 2011.

[27] A. Mihailovic, M. Simeunovi, N. Leki, and M. Pejanovi, "A strategy for deploying diverse sensor-based networks as an evolution towards integrated Internet of Things and Future Internet," pp. 23-26, 2014.

[28] J. Zhou, T. Leppanen, E. Harjula, M. Ylianttila, T. Ojala,
C. Yu, and H. Jin, "CloudThings: A common architecture for integrating the Internet of Things with Cloud Computing," Proc. 2013 IEEE 17th Int. Conf. Comput. Support. Coop. Work Des. CSCWD 2013, pp. 651-657, 2013.

[29] M. I. Alam, M. Pandey, and S. S. Rautaray, "A Comprehensive Survey on Cloud Computing," I.J. Inf. Technol. Comput. Sci. Inf. Technol. Comput. Sci., vol. 02, no. 02 , pp. 68-79, 2015.

[30] T. Liu, "Application of Cloud Computing in the Emergency Scheduling Architecture of the Internet of Things," 2015.

[31] T. Y. Wu, G. H. Liaw, S. W. Huang, W. T. Lee, and C. C. Wu, "A GA-based mobile RFID localization scheme for internet of things," Pers. Ubiquitous Comput., vol. 16, no. 3, pp. 245-258, 2012.

[32] N. Bevan, "Measuring usability as quality of use," Softw. Qual. J., vol. 4, no. 2, pp. 115-130, 1995.

[33] N. Zeni and L. Mich, "Usability issues for systems supporting requirements extraction from legal documents," 2014 IEEE 7th International Workshop on Requirements Engineering and Law, RELAW 2014 Proceedings, 2014.

[34] C. Eliasson, M. Fiedler, and I. Jørstad, "A criteria-based evaluation framework for authentication schemes in IMS," Proc. - Int. Conf. Availability, Reliab. Secur. ARES 2009, pp. 865-869, 2009.

[35] O. Gioug, K. Dooyeon, K. Sangil, and R. Sungyul, “A quality evaluation technique of RFID middleware in ubiquitous computing," Proc. - 2006 Int. Conf. Hybrid Inf. Technol. ICHIT 2006, vol. 2, pp. 730-735, 2006.

[36] V. Nassar, "Common criteria for usability review," Work, vol. 41, no. SUPPL.1, pp. 1053-1057, 2012.

[37] U. O. Nwokedi, B. A. Onyimbo, and B. B. Rad, "Usability and Security in User Interface Design: A Systematic Literature Review," Int. J. Inf. Technol. Comput. Sci., vol. 8, no. 5, pp. 72-80, 2016.

[38] T. Jokela, "Assessments of usability engineering processes: experiences from experiments," 36th Annu. Hawaii Int. Conf. Syst. Sci. 2003. Proc., p. 9 pp., 2003.

[39] C. Prehofer, "From the internet of things to trusted apps for things," Proc. - 2013 IEEE Int. Conf. Green Comput. Commun. IEEE Internet Things IEEE Cyber, Phys. Soc. Comput. GreenCom-iThings-CPSCom 2013, pp. 20372042, 2013.

[40] N. Maalel, E. Natalizio, A. Bouabdallah, P. Roux, and M. Kellil, "Reliability for emergency applications in internet of things," Proc. - IEEE Int. Conf. Distrib. Comput. Sens. Syst. DCoSS 2013, pp. 361-366, 2013.

[41] N. Nikmehr, "Content y Usability,"pp. 347-351, 2008.

[42] S. Jimenez-Fernandez, P. De Toledo, and F. Del Pozo, "Usability and interoperability in wireless sensor networks for patient telemonitoring in chronic disease management," IEEE Trans. Biomed. Eng., vol. 60, no. 12, pp. 3331-3339, 2013.

[43] R. A. Canessane and S. Srinivasan, "A Frameworkfor Analy sing the Sy stem Quality," pp. 1111-1115, 2013.

[44] I. Lee and K. Lee, "The Internet of Things (IoT): Applications, investments, and challenges for enterprises," Bus. Horiz., vol. 58, no. 4, pp. 431-440, 2015.

[45] A. J. Jara, Y. Bocchi, and D. Genoud, "Social internet of things: The potential of the internet of things for defining human behaviours," Proc. - 2014 Int. Conf. Intell. Netw. Collab. Syst. IEEE INCoS 2014, pp. 581-585, 2015.

[46] P. Patel, A. Pathak, T. Teixeira, Val, \#233, and R. Issarny, "Towards application development for the internet of 
things," Proc. 8th Middlew. Dr. Symp., pp. 1-6, 2011.

[47] J. Kim and J.-W. Lee, "OpenIoT: An open service framework for the Internet of Things," 2014 IEEE World Forum Internet Things, pp. 89-93, 2014.

[48] W. Zhang, "Study about IOT' s Application in 'Digital Agriculture' Construction," Inf. Sci. (Ny)., pp. 2578-2581, 2011.

[49] Y. Xu, Y. Wang, X. Gao, and S. Zhang, "Product Development Process Improvement Approach Based on Benchmarking," Electronics, pp. 1-4, 2010.

[50] M. H. Abdallah, "A quality assurance model for an information system development life cycle," Int. J. Qual. Reliab. Manag., vol. 13, no. 7, pp. 23-35, 1996.

[51] L. Yong-Fei and T. Li-Qin, "Comprehensive Evaluation Method of Reliability of Internet of Things," 2014 Ninth Int. Conf. P2P, Parallel, Grid, Cloud Internet Comput., pp. 262-266, 2014.

[52] S. Thomas, G.E, "Dual RFID-ZigBee Sensor enable NFC application for internet of things," 2012. [Online]. Available: http://www.electronicssourcing. com/2012/03/28/dual-rfid-zigbee-sensorsenable-nfc-applications-for-the-internet-of-things/.

\section{Authors' Profiles}

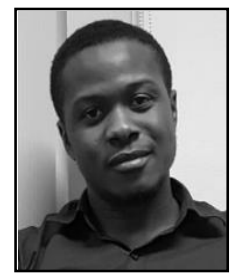

Michael Onuoha Thomas received his B.Sc. Degree in Computer Science from Caritas University, Nigeria in 2012 and currently pursuing his M.Sc. Degree in Software Engineering at Asia Pacific University of Technology and Innovation under Staffordshire University franchised program. His research interests include software security, reliability engineering, software development process modeling, software project management, fog computing, cloud computing and internet of things.

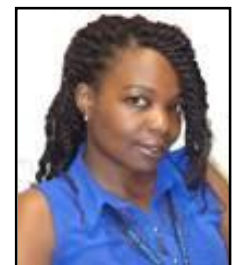

Beverly Amunga Onyimbo awarded Bachelor Degree from Kabarak University, Kenya in 2012, currently pursuing her M.Sc. Degree in Software Engineering at Asia Pacific University of Technology and Innovation under Staffordshire University franchised program. Her current research interests include User Experience and User Interface (UX \& UI) Design, Mobile Application Development, Human-Computer Interaction, Requirements Engineering, Computer Communication Networks, Artificial Intelligence, Fog Computing, Internet of things and Information Security .

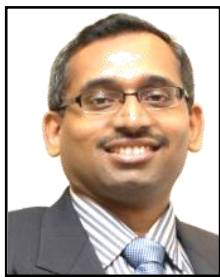

Rajasvaran Logeswaran studied his B.Eng (Hons) Computing at Imperial College London, United Kingdom, and completed his M.Eng.Sc. as well as Ph.D. at Multimedia University, Malaysia. He is a Novell Certified Linux Professional, and a certified IC Digital Citizen and trainer. His areas of interests include multimedia data processing, data compression, neural networks, natural user interfaces and big data, with over a hundred publications in books, peer-reviewed journals and international conference proceedings. He has been a recipient of several scholarships, including Telekom Malay sia, the JCS 75th Anniversary Scholar, the Brain Gain Malaysia international fellowship \& postdoctoral programme, as well as the Brain Korea21 post-doctoral grant. A Senior Member of the IEEE, he is the Secretary of the IEEE Signal Processing Society Malaysia chapter and a reviewer of numerous journals and conferences.

How to cite this paper: Michael Onuoha Thomas, Beverly Amunga Onyimbo, Rajasvaran Logeswaran, "Usability Evaluation Criteria for Internet of Things", International Journal of Information Technology and Computer Science(IJITCS), Vol.8, No.12, pp.10-18, 2016. DOI: 10.5815/ijitcs.2016.12.02 